importanza" sul grande impero di Vijayanagar (102), "primissime" quelle riferite sul Borneo (175). Per la descrizione della moschea di Medina, Musacchio scrive che "Varthema si rivela uno straordinario osservatore" (34). Nelle descrizioni il curatore vede spesso prove di acutezza dell' antico viaggiatore e, come nel capitolo riguardante la navigazione a Calicut, nota che egli "si rivela un osserva-tore acuto e preciso" (123). Nella città di Tenasserim si legge ad esempio che "dopo la morte del marito la donna si brucia" (152); in nota Musacchio fa riferimento ad altri viaggiatori che hanno riferito tale costume, ma rileva che "la descrizione più vivida è ancora quella del Varthema che sembra, con il suo abituale acume, proporre delle soluzioni ai quesiti che il lettore si viene ponendo" (154).

In alcune descrizioni sembra che il Varthema si rifaccia a modelli precedenti; ciononostante riesce sempre a lasciare quella traccia che rende la descrizione tutta 'varthemiana'. È il caso del capitolo 19 in cui tratta dell'unicorno. Musacchio sottolinea il fatto che il Varthema non è propenso ad inventare, eppure "la descrizione è così particolareggiata da rendere difficile un atteggiamento totalmente scettico sullo spettacolo visto e descritto dal nostro viaggiatore" (47). A volte il Varthema impiega termini poco chiari, come nel caso della descrizione del pepe (125), ma più spesso i termini sono precisi e vivaci come nella descrizione del sapore del frutto del "jack". Musacchio scrive che "nel tentativo di descrivere la peculiarità del gusto di questo frutto, [Varthema] si lancia in un multiforme, quasi pirotecnico, paragone con una rimarchevole varietà di frutti rari" (127). L'omologazione del nuovo, filtrato dalle categorie già possedute, ci ripropone il problema affrontato da quegli antichi viaggiatori: come tradurre in parole la massa di impressioni che avevano avuto davanti a sé.

L'Itinerario è corredato di note utilissime e sempre affascinanti per l'estensione e la documentazione erudita. Il volume si presenta in veste tipograficamente curatissima (peccato che sia privo di un indice dei nomi); nel complesso costituisce un solido contributo che colma degnamente una lacuna nella viaggistica cinquecentesca e testimonia con chiarezza la cultura di Musacchio e le sue profonde conoscenze in questo campo.

\title{
ENRICO VICENTINI
}

Victoria University in the University of Toronto, CRRS

Maria-Luisa Minio-Paluello. La "Fusta dei Matti". Firenze: giugno 1514. Introd. di Franco Cardini. Firenze: Franco Cesati, 1990. Pp. 197.

Il 22 giugno 1514, due giorni prima della festa civica e religiosa del patrono san Giovanni, si assisteva a Firenze ad un insolito trionfo. Un carro in forma di nave (fusta) percorreva le strade della città, portando un carico di buffoni (matti), e circondato da una schiera di "diavoli". Capitano di questa Narrenshiff era Maestro Antonio di Pierrozzo da Vespignano, cappucciaio, un uomo descritto nei documenti come "un poco isciemo ma... verboso e piacevole". Strada facendo, il carro con il suo corteo trionfale si imbatteva nel 
portatore di lana Giovanni Tancredi, "più scioccho assai di Maestro Antonio sopraddetto". Alcuni dei "diavoli" che accompagnavano il carro catturavano il povero Tancredi e, calato un corbello dalla fusta, lo facevano issare a bordo, dove veniva messo "a remo" e bastonato.

Questa festa, allestita "per dare piacere al popolo", è il soggetto del presente studio. E con ragione: la Minio-Paluello intuisce chiaramente, come pure intuirono i Cardinali venuti da Roma alcuni giorni prima, che si trattava di più di un semplice episodio burlesco. Come tanti altri "trionfi", anche questo aveva un "lato oscuro . . . [una] minaccia sovversiva" (17) che tradiva un sottofondo politico espresso tramite le figure emblematiche del carro trionfale. Lo studio della Minio-Paluello, quindi, esamina questo insolito episodio burlesco come fosse l'emblema di una ben precisa situazione politica. La restaurazione medicea, il governo di Lorenzo di Piero de' Medici, la politica estera di Leone X vengono presi di mira e, per così dire, beffati dai marinai e dai passeggeri della fusta.

Il saggio si divide in due parti. Nella prima la studiosa cerca di rintracciare le fonti di una simbologia del folle e della nave dei folli nella tradizione festiva fiorentina. Nella seconda, invece, esamina il "canovaccio storico, che in certo modo si è fatto testo (tessuto) della festa" (156) Il tutto viene fatto secondo una lettura "relativamente soggettiva . . . proposta non solo nel punto d'arrivo, ma nel suo costruirsi" (22). Il lettore, quindi, segue lo svilupparsi, l'ampliarsi e l'approfondirsi dello studio tramite i labirinti dell'effimero storico, della simbologia polivalente che costituisce e colora il trionfo rinascimentale: a fine tragitto, tuttavia, si trova alle prese con molte congetture, ma poche risposte. Franco Cardini, nella sua introduzione, aveva già avvisato che "molti restano ancora gli interrogativi" e aveva indicato che il valore di questo studio risiedeva non nelle risposte, ma nelle "questioni . . . qui poste e . . a affrontate" (7). Il saggio, dunque, diventa una valida indagine su fatti, simboli e giochi politici desunti da una storia che, anche allora, si rivelava velandosi.

Tuttavia per alcuni aspetti il lavoro della Minio-Paluello avrebbe potuto essere migliorato. Così, dato che non tutti i lettori possono conoscere l'episodio, sarebbe stato utile avere già all'inizio un riassunto chiaro e complessivo degli avvenimenti da esaminare; l'arrivo a Firenze dei Cardinali romani, per esempio, coglie il lettore del tutto di sorpresa, come fosse un asso nella manica della studiosa (139 sgg.). Inoltre le tredici illustrazioni alla fine del volume sono interessanti, ma per lo più gratuite e superflue: molte infatti non fanno parte di un approfondito esame scientifico vis à vis la tradizione o l'avvenimento che sono il soggetto dello studio. Anche il linguaggio avrebbe potuto essere snellito: il tono colloquiale tende alle ripetizioni e dà luogo a congetture. Alcune interpretazioni, come quella che associa il solstizio di san Giovanni con quello di "Natale-Carnevale" (58), oppure l'analisi dell'appellativo Carafulla (63), sembrano forzate. Infine, anche ad una prima lettura sono balzati all'occhio una ventina di errori tipografici non inclusi nell'Errata corrige. Un lavoro di lima più attento avrebbe agevolato la lettura e dato più forza al discorso.

KONRAD EISENBICHLER

Victoria College, University of Toronto 